\title{
Peritoneal dialysis as successful treatment in patient with end-stage congestive heart failure due to endomyocardial fibrosis
}

\author{
Francesco Sbrana ${ }^{a}$, Antonio Cioni ${ }^{b}$, Vladyslav Chubuchny ${ }^{a}$, Claudia Taddeia, \\ Roberto Bigazzi ${ }^{b}$, Emilio Maria Pasanisia \\ a Fondazione Toscana Gabriele Monasterio, Pisa, Italy \\ ${ }^{\text {b }}$ U.O. di Nefrologia e Dialisi, Dipartimento di Medicina clinica e ad indirizzo specialistico - Ospedale di Livorno, Livorno, Italy
}

\section{ARTICLE INFO}

Article history:

Received: 23. 10. 2017

Accepted: 8. 4. 2018

Available online: 27. 4. 2018

Since 2011 a 70-year-old Caucasian woman with precedent diagnosis, after a multimodality imaging evaluation, ${ }^{1}$ of endomyocardial fibrosis (EMF) was followed for progressive heart failure.

At the diagnosis she presented 4 major diagnostic criteria (endomyocardial plaques, obliteration of the LV apex, LV thrombosis, moderate-to-severe mitral regurgitation) and 2 minor criteria (restrictive filling pattern, enlarged left atrium) of EMF. ${ }^{2}$ She underwent anuloplasty of the aortic and mitral valves and cardiac resynchronization therapy. After one year the patient's conditions were stable. ${ }^{1}$ In the following six years she was admitted more times to our Institute for heart failure (NYHA class III-IV) and showed a progressive increase of brain natriuretic peptide (BNP) (Fig. 1A).

Respect to the 2D echocardiography performed after mitral valve surgery (Fig. 1B) the apical 4-chamber view, tricuspidal regurgitation and inferior vena cava (IVC) re-

Fig. 1 - (A) Time course of brain natriuretic peptide (BNP). (B) 2D echocardiography after mitral valve surgery: in the apical 4-chamber view near to normal right ventricular systolic function (FAC = $35 \%$, TAPSE $=15 \mathrm{~mm}$ ), and moderate-to-severe tricuspidal regurgitation; dilated inferior vena cava (IVC) with respiratory collapse $50 \%$. (C) $2 \mathrm{D}$ echocardiography in right ventricular failure: in the apical 4-chamber view presence of moderate right ventricular systolic dysfunction (FAC $=18 \%$, TAPSE $=14 \mathrm{~mm}$ ) and severe tricuspidal regurgitation; dilated inferior vena cava (IVC) with no respiratory collapse. (D) 2D echocardiography after the beginning of peritoneal dialysis: in the apical 4-chamber view normal right ventricular systolic function (FAC $=39 \%$, TAPSE $=16 \mathrm{~mm}$ ), and moderate tricuspidal regurgitation; normal inferior vena cava (IVC) with respiratory collapse $>50 \%$.

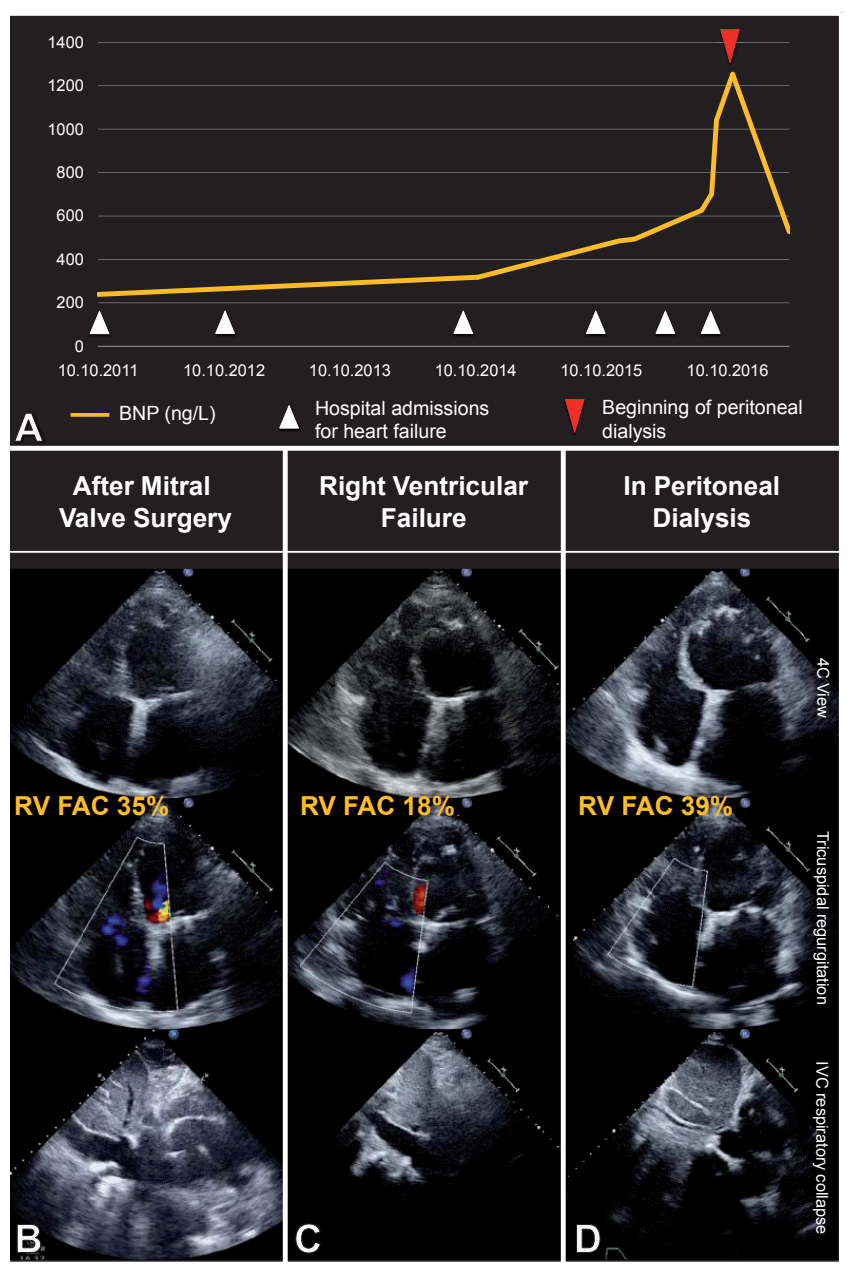

Address: Francesco Sbrana, MD, Fondazione Toscana Gabriele Monasterio, Via Moruzzi 1, 56124 Pisa, Italy, e-mail: francesco.sbrana@ftgm.it DOI: 10.1016/j.crvasa.2018.04.001

(C) 2019, ČKS. 
spiratory collapse showed a progressive worsening (Fig. 1C). Due to the end-stage congestive heart failure peritoneal dialysis was started and just after 6 months patient's conditions (NYHA class IIb - stable decrease in weight of $7 \mathrm{~kg}$ ), BNP (Fig. 1A) and 2D echocardiography were improved (Fig. 1D).

EMF is characterized by ventricular hypertrophy and diffuse endocardial thickening due to the deposition of collagen and elastin, with secondary calcification ${ }^{3}$ and has a progressive natural history that frequently leads to refractory heart failure.

Nowadays, peritoneal dialysis is a therapeutic alternative in patients with advanced congestive heart failure ${ }^{4}$ and represents an alternative therapeutic option in patient not-suitable for cardiac transplantation. ${ }^{5}$

In this patient peritoneal dialysis provides, also in the EMF, a safe effective means for dehydrating patients with end-stage congestive heart failure improving patients' cardiac function, symptoms and quality of life.

\section{Conflict of interest}

The authors declare that they have no conflict of interest.

\section{Funding}

None.

\section{Ethical statement}

All procedures performed in studies involving human participants were in accordance with the ethical standards of the institutional and/or national research committee and with the 1964 Helsinki declaration and its later amendments or comparable ethical standards.

\section{Informed consent}

The patient's consent has been obtained.

\section{References}

1. Sbrana F, Coceani M, Masci PG, et al. Calcified apical cardiomyopathy: a rare form of endomyocardial fibrosis. J Cardiovasc Med (Hagerstown) 2015;16(Suppl 2):S79-S80.

2. Mocumbi AO, Ferreira MB, Sidi D, Yacoub MH. A population study of endomyocardial fibrosis in a rural area of Mozambique. N Engl J Med 2008;359:43-49.

3. Aras D, Topaloglu S, Demirkan B, et al. Porcelain heart: a case of massive myocardial calcification. Int J Cardiovasc Imaging 2006;22:123-126.

4. Núñez J, González M, Miñana G, et al. Continuous ambulatory peritoneal dialysis as a therapeutic alternative in patients with advanced congestive heart failure. Eur J Heart Fail 2012;14:540-548.

5. Díez-Villanueva P, Sousa I, Fernández-Avilés F. Advanced heart failure due to endomyocardial fibroelastosis. Rev Clin Esp 2016;216:230. 